\title{
Age Is a Determinant of Leukocyte Infiltration and Loss of Cortical Volume after Traumatic Brain Injury
}

\author{
Catherine P. Claus ${ }^{\mathrm{a}}$ Kyoko Tsuru-Aoyagi ${ }^{\mathrm{a}}$ Hita Adwanikar ${ }^{\mathrm{a}}$ Breset Walker $^{\mathrm{b}}$ \\ William Whetstone $^{\mathrm{b}}$ Linda J. Noble-Haeusslein ${ }^{\mathrm{a}, \mathrm{c}}$ \\ Departments of ${ }^{a}$ Neurological Surgery, ${ }^{b}$ Emergency Medicine and ${ }^{c}$ Physical Therapy and Rehabilitative Science, \\ University of California, San Francisco, Calif., USA
}

\section{Key Words}

Inflammation - Traumatic brain injury • Developing brain •

Cell death

\begin{abstract}
There is increasing evidence that the inflammatory response differs in the injured developing brain as compared to the adult brain. Here we compared cerebral blood flow and profiled the inflammatory response in mice that had been subjected to traumatic brain injury (TBI) at postnatal day (P)21 or at adulthood. Relative blood flow, determined by laser Doppler, revealed a $30 \%$ decrease in flow immediately after injury followed by prominent hyperemia between 7 and 35 days after injury in both age groups. The animals were euthanized at 1-35 days after injury and the brains prepared for the immunolocalization and quantification of CD45-, GR-1-, CD4- and CD8-positive (+) cells. On average, the number of CD45+ leukocytes in the cortex was significantly higher in the P21 as compared to the adult group. A similar trend was seen for GR-1+ granulocytes, whereas no age-related differences were noted for CD4+ and CD8+ cells. While CD45+ and GR-1+ cells in the P21 group remained elevated, relative to shams, over the first 2 weeks after injury, the adult group showed a time course limited to the first 3 days after injury. The loss of ipsilateral cortical volumes at 2 weeks after injury
\end{abstract}

\section{KARGER}

Fax +41613061234 E-Mail karger@karger.ch www.karger.com
(C) 2010 S. Karger AG, Basel

Accessible online at: www.karger.com/dne was significantly greater in the adult relative to the P21 group. While the adult group showed no further change in cortical volumes, there was a significant loss of cortical volumes between 2 and 5 weeks after injury in the P21 group, reaching values similar to that of the adult group by 5 weeks after injury. Together, these findings demonstrate age-dependent temporal patterns of leukocyte infiltration and loss of cortical volume after TBI.

Copyright $\odot 2010$ S. Karger AG, Basel

\section{Introduction}

Traumatic brain injury (TBI) results in significant morbidity and mortality of the pediatric population [Levin et al., 1982; Fife and Jagger, 1984; Frankowski, 1986; Luerssen et al., 1988; Kraus et al., 1990; Philip et al., 2009]. Brain-injured children younger than 4 years of age exhibit more severe cognitive and motor deficits than older children [Durkin et al., 1998]. While the biologic factors that contribute to this vulnerability are unclear, studies suggest that inadequate antioxidant reserves may be a determinant of these outcomes [Ditelberg et al., 1996;

C.P.C. and K.T.-A. contributed equally to this paper.
Linda J. Noble-Haeusslein, $\mathrm{PhD}$

Department of Neurological Surgery, University of California, San Francisco

521 Parnassus Avenue, Room C224

San Francisco, CA 94143-0112 (USA)

Tel. +1 415476 4850, Fax +1 415476 5634, E-Mail Linda.Noble@ ucsf.edu 
Mischel et al., 1997; Ferriero, 2001; Fan et al., 2003; TsuruAoyagi et al., 2009].

Here we consider the possibility that inflammation may likewise contribute to the vulnerability of the injured, developing brain. There is evidence that the magnitude of inflammation may be age dependent. In response to acute exposure to cytokines, the kinetics of leukocyte recruitment vary according to age, with the adult brain showing fewer leukocytes than 1- to 3-week-old animals [Lawson and Perry, 1995; Anthony et al., 1997]. Such findings have led to the hypothesis that there is a 'window of susceptibility' where the developing brain is less refractory to inflammatory stimuli than the adult brain [Anthony et al., 1998].

There is evidence to support the pathogenicity of leukocytes in the acutely injured brain. Leukocytes, including neutrophils, release free radicals, proteases and proinflammatory cytokines, all of which can promote tissue damage [Owen and Campbell, 1999; Lee and Downey, 2001; Kawabata et al., 2002]. The recruitment of leukocytes into the traumatized adult brain [Schoettle et al., 1990; Biagas et al., 1992; Soares et al., 1995; Hartl et al., 1997] occurs in response to the activation of complement [Kaczorowski et al., 1995] and coincides with a disruption of the blood-brain barrier [Soares et al., 1995]. In the developing brain, the cause and effect of leukocyte accumulation and outcome in an immature TBI model have not been demonstrated.

We have previously characterized reproducible models of traumatic injury to the developing and adult murine brains and have shown predictable patterns of cortical and subcortical neuronal vulnerability [Igarashi et al., 2001; Tong et al., 2002; Tsuru-Aoyagi et al., 2009]. Here we profile leukocyte infiltration and longer-term changes in cortical mantle volume in each of these models and demonstrate age-dependent differences in these parameters. Such findings highlight unique differences in how the developing and adult brains respond to a traumatic injury, and they underscore the need to further investigate the mechanisms that contribute to age-dependent pathogenesis.

\section{Materials and Methods}

\section{Surgical Procedures}

Male postnatal day (P)21 C57BL/6 mice were anesthetized with $1.25 \%$ 2,2,2-tribromoethanol, diluted in isotonic saline at $0.02 \mathrm{ml} / \mathrm{g}$ body weight. Adult male C57BL/6 mice ( 3 months old) were anesthetized with a higher concentration of $2.5 \%$ 2,2,2-tribromoethanol solution. All animals were placed on a water-cir- culating heating pad throughout the surgery and recovery. All animals fully recovered within $3 \mathrm{~h}$ after surgery. Each animal was placed in a stereotaxic frame (David Kopf Instruments, Tujunga, Calif., USA) for surgery.

After a midline skin incision, the soft tissues were reflected and blood flow measurements initiated using a laser Doppler probe. A circular craniotomy, $5.0 \mathrm{~mm}$ in diameter, was made with a micro-drill between bregma and lambda with a medial edge of the craniotomy $0.5 \mathrm{~mm}$ lateral to the midline. In the injured group, each animal was then positioned in a stereotaxic frame of the injury device and was subjected to a controlled cortical impact injury [Dixon et al., 1991; Igarashi et al., 2001; Tong et al., 2002; Fan et al., 2003], using a convex impactor tip that was $3.0 \mathrm{~mm}$ in diameter and oriented perpendicular to the surface of the brain. The injury was generated using the following parameters: $4 \mathrm{~m} / \mathrm{s}$ velocity, $1.0 \mathrm{~mm}$ depth of penetration and a sustained depression of $150 \mathrm{~ms}$. Sham-operated animals underwent the same surgical procedures with the exception of the cortical impact. In both the sham and brain-injured groups, the scalp was then closed with sutures. Each animal was given $1.0 \mathrm{ml}$ of isotonic saline subcutaneously after the operation to prevent dehydration. The shamoperated animals were euthanized at 1 day, 14 days and 5 weeks after surgery. The brain-injured animals were euthanized at 1, 3, 7 and 14 days and 5 weeks after injury.

\section{Blood Flow Measurements}

A laser Doppler device (Laserflo BPM2; Vasamedics, USA) was used to measure relative cerebral blood flow (CBF). Following the craniotomy, blood flow was measured from the surface of the brain. When taking measurements, care was taken to avoid grossly visible blood vessels. Following cortical impact and again prior to euthanasia, the probe was returned to the same position and a measurement of blood flow was again taken from the surface of the brain.

The change in blood flow is expressed as a percentage of the preinjury CBF level: [(CBF at a given time point - $\mathrm{CBF}$ before injury)/CBF before injury $\times 100]$. Blood flow was analyzed using two-way ANOVA (to compare the adult vs. P21 trends in the change in blood flow) using Stata version 8.0 (StataCorp, College Station, Tex., USA), followed by Sidak's multiple comparisons to compare changes over days within each group.

\section{Immunocytochemistry}

Animals ( $\mathrm{n}=3-5 /$ group) were perfused through the heart with $50 \mathrm{ml}$ of $4 \%$ paraformaldehyde in $0.1 \mathrm{M}$ phosphate-buffered saline (PBS), at a $\mathrm{pH}$ of 7.4. The brains were removed, fixed in $4 \%$ paraformaldehyde for $3.5 \mathrm{~h}$ and cryoprotected in sucrose (30\% in PBS) for $48 \mathrm{~h}$. The cryoprotected brains were embedded in tissue freezing medium (Triangle Biomedical Sciences, Durham, N.C., USA) and frozen at $-70^{\circ} \mathrm{C}$. Frozen sections, $20 \mu \mathrm{m}$ in thickness, were cut on a cryostat in the coronal plane and collected on slides ( 2 sections/slide). Sections of brain were processed for conventional immunocytochemistry. Multiple antibodies were tested using a different series of slides from each brain, comprising every 10th slide. The antibodies used were as follows: leukocytes (antiCD45; Caltag M6404; 1:250), neutrophils (GR-1; Caltag RM3000; 1:500), 'helper' T cells (anti-CD4; Serotec MCA1108G; 1:150) and cytotoxic T cells (anti-CD8; Serotec MCA612; 1:150). Tissue from both groups at all time points was stained with these antibodies ( $n=3-5 /$ group). All primary antibodies were diluted in $2 \%$ rabbit 
serum (RS)/0.2\% Triton X-100 (TX)/BSA (2\% RS/TX/BSA). The sections were first rehydrated in $0.1 \mathrm{M} \mathrm{PBS}$ ( $\mathrm{pH} 7.4$ ) for $5 \mathrm{~min}$. Secondly, they were incubated in $0.003 \%$ hydrogen peroxide $\left(\mathrm{H}_{2} \mathrm{O}_{2}\right)$ in $0.1 \mathrm{M}$ PBS for $10 \mathrm{~min}$ to quench any endogenous peroxidase activity, and then incubated in each of the following solutions for the time indicated: $2 \% \mathrm{RS} / \mathrm{TX} / \mathrm{BSA}$ ) in $0.1 \mathrm{M}$ PBS for $5 \mathrm{~min} ; 10 \%$ $\mathrm{RS} / 0.2 \% \mathrm{BSA} / 0.2 \% \mathrm{TX}$ in $0.1 \mathrm{M}$ PBS for $20 \mathrm{~min}$; primary antibody for $2 \mathrm{~h}$ at $4^{\circ} \mathrm{C}$; PBS 5 times $3 \mathrm{~min}$; rabbit anti-rat IgG (1:200 2\% RS/TX/BSA; Vector BA-4001) for 1 h; PBS 5 times 3 min; avidinbiotin-horseradish-peroxidase complex (Vectastain ABC Kit; 1:100 in PBS; Vector Labs) for $30 \mathrm{~min}$, and PBS 5 times $3 \mathrm{~min}$. The final reaction product was visualized using 0.05\% 3,3-diaminobenzidine tetrachloride as the chromogen in the presence of $0.02 \% \mathrm{H}_{2} \mathrm{O}_{2}$ for $10 \mathrm{~min}$. The immunostained sections were then dehydrated in graded alcohols, cleared in Hemo-De (Fisher Scientific Inc., Pittsburgh, Pa., USA), and coverslipped with Permount (Fisher Scientific). Immunocytochemical controls, completed on adjacent sections of brain, consisted of the same reaction procedures in the absence of primary antibody.

\section{Localization of Irreversibly Damaged Cells}

Terminal deoxynucleotidyl-transferase-mediated dUTP nick end labeling (TUNEL) was used to assess irreversible cell damage in all animals at 1,3 and 7 days after injury ( $n=3-5$ /group). The colorimetric TdT-FragEL DNA Fragmentation Detection Kit (Oncogene Research Products; product No. QIA33) was used in this study. The staining methodology was executed according to their provided instructions. A positive and a negative control were run with each batch of slides. Following staining, the slides were coverslipped with Permount mounting medium (Fisher Scientific).

Regional Cell Counting and Estimation of Cortical Volumes

Cell counting and estimation of volumes were conducted in a masked fashion. The number of GR-1+, CD8+, CD4+ and CD45+ cells were determined on 4 equally spaced coronal sections from both groups ( $\mathrm{n}=3-5$ /group) at 1, 3, 7 and 14 days and 5 weeks after injury. TUNEL+ cells were counted on 4 equally spaced coronal sections from each brain for both groups ( $n=3-5$ /group) at 1,3 and 7 days after injury. These serially spaced sections were each separated by $400 \mu \mathrm{m}$ (every 20th section) to span a range of 1,600 $\mu \mathrm{m}$. The first section was taken from the same anatomical plane (bregma $-1.46 \mathrm{~mm}$; anterior to injury) in each animal, as well as the last section (bregma $-3.06 \mathrm{~mm}$; posterior to injury) in order to include the site of maximal injury in all cases. The number of positively stained cells was determined within the contoured region of the remaining ipsilateral cortical mantle. To quantify positively stained cells, a contour was drawn using the Neurolucida software (MicroBrightField Inc., Williston, Vt., USA), and all positively stained cells were counted within these defined boundaries. All distinctly stained cells were identified at $\times 60$ magnification using Neurolucida in the ipsilateral and contralateral hemispheres of the brain-injured mice and sham controls. There was minimal positive staining on the contralateral side (data not shown). A cell was considered for counting when it had a clearly defined pattern of membrane staining. Partial profiles were not counted, thus leading to an underestimate of the number of cells present per section. Adjacent sections were used in the analysis as an alternative in the event of tissue damage. The cell counts were expressed for a region of interest as the mean number of cells per section.
Cortical volumes were estimated at 14 and 35 days after injury in both age groups ( $n=5 /$ group). Four serial sections stained with cresyl violet were used for quantification. In each brain, the most anterior section was selected at bregma $-1.46 \mathrm{~mm}$, and each section was separated by $400 \mu \mathrm{m}$ [Paxinos and Watson, 1986]. The spared areas of the ipsilateral cortical mantle, as identified by cresyl violet staining, were outlined and the volume was computed using the Cavalieri method by multiplying the sum of the profile areas of the region of interest on all sections with the distance between the sections [Gundersen and Jensen, 1987; Reed and Howard, 1998].

\section{Results}

\section{Relative Cerebral Blood Flow Is Similar between} Developing and Adult Brains after TBI

We first determined if blood flow after TBI showed age-dependent differences. The effects of age at time of injury and time after injury on relative blood flow were evaluated by two-way ANOVA (fig. 1). There was no significant interaction between these factors $(p=0.13)$, thus the interaction term was dropped and a main effects analysis performed. Main effects analysis revealed a statistically significant effect $(\mathrm{p}<0.001)$ for time after injury, but not a significant difference for age $(p=0.76)$. Multiple comparisons among the days after injury using Sidak's method showed that blood flow immediately after injury was significantly lower relative to all other time points in the P21 group ( $p<0.001)$. Blood flow in the adult brain remained depressed for the first 3 days after injury. Thereafter, blood flow was significantly elevated relative to that measured immediately after injury $(\mathrm{p}<$ 0.002 ).

\section{The Temporal Pattern of Leukocyte Recruitment Is Age Dependent}

Next we compared leukocyte recruitment in the injured P21 and adult brains at 1-35 days after injury. CD45+ cells were noted throughout the injured ipsilateral cortex as well as in the hippocampus in both age groups (fig. 2). A majority of these cells were localized adjacent to the site of cortical injury, and the phenotype varied from round cells bearing no processes (fig. 2b, e) to cells with short processes (fig. 2c, f).

The distribution of GR-1+ cells was similar to that of CD $45+$ cells in both the developing (fig. 3 ) and adult brains (fig. 4), appearing most prominently in the cortex but also within the underlying hippocampus. To confirm the identity of these cells, adjacent sections stained with hematoxylin and eosin revealed polylobulated nuclei, a feature consistent with that of neutrophils (fig. 3d, e, i, j, 4d, e, i, j). 
Fig. 1. Relative cerebral blood flow in the injured P21 (a) and adult brain (b). There is a statistically significant time-after-injury effect, but not a significant difference for age. ${ }^{*} \mathrm{p}<0.002$; ${ }^{* *} \mathrm{p}<0.001$. a Multiple comparisons among the days after injury using Sidak's method shows that blood flow immediately after injury (day 0) is significantly lower relative to other time points for the P21 group. b Blood flow in the adult brain remains depressed for the first 3 days after injury. Thereafter, blood flow is significantly elevated relative to day 0 .
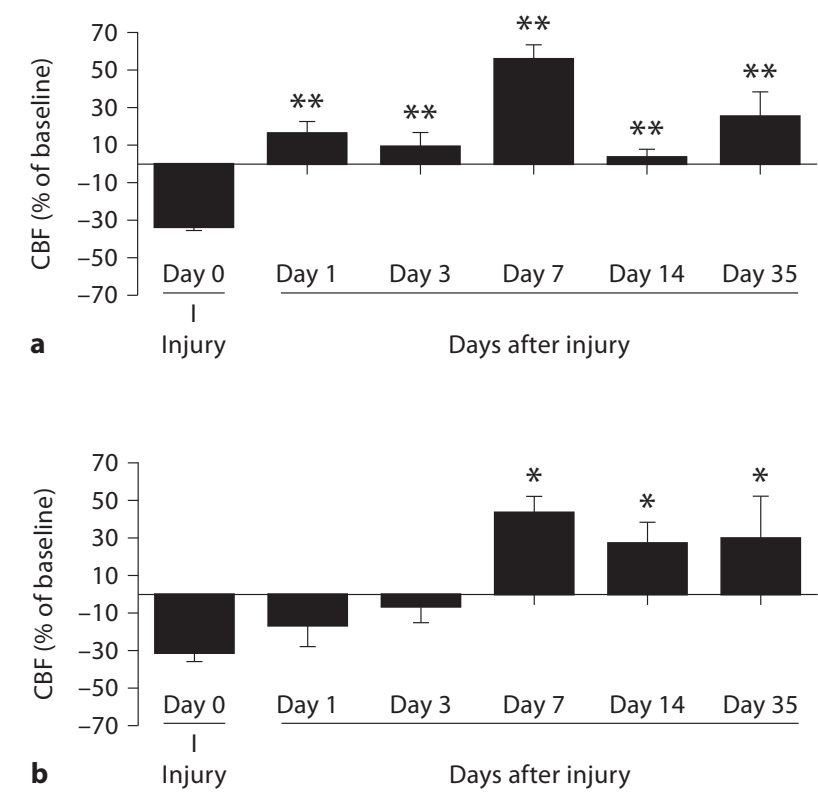
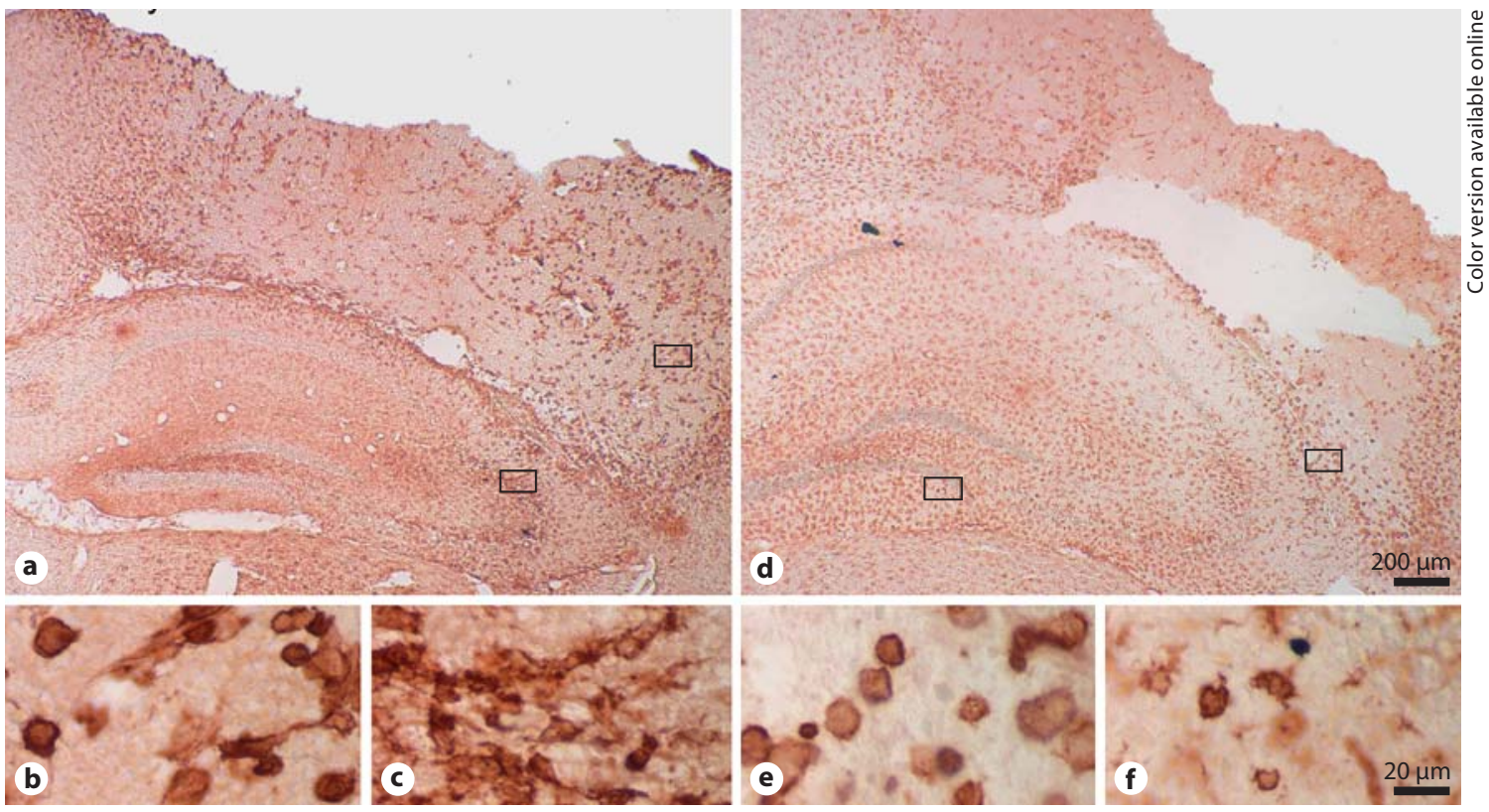

Fig. 2. CD45+ cells in the acutely injured brain. Localization of the panleukocytic marker CD45 in the P21 $(\mathbf{a}-\mathbf{c})$ and adult (d-f) brains at 3 days after injury. Boxes: locations of enlarged images of the cortex (b, e) and hippocampus (c, f). Note the prominent accumulation in both the cortex and hippocampus of both age groups. Phenotypes of these cells include round cells with no processes as well as cells bearing a few short processes. 

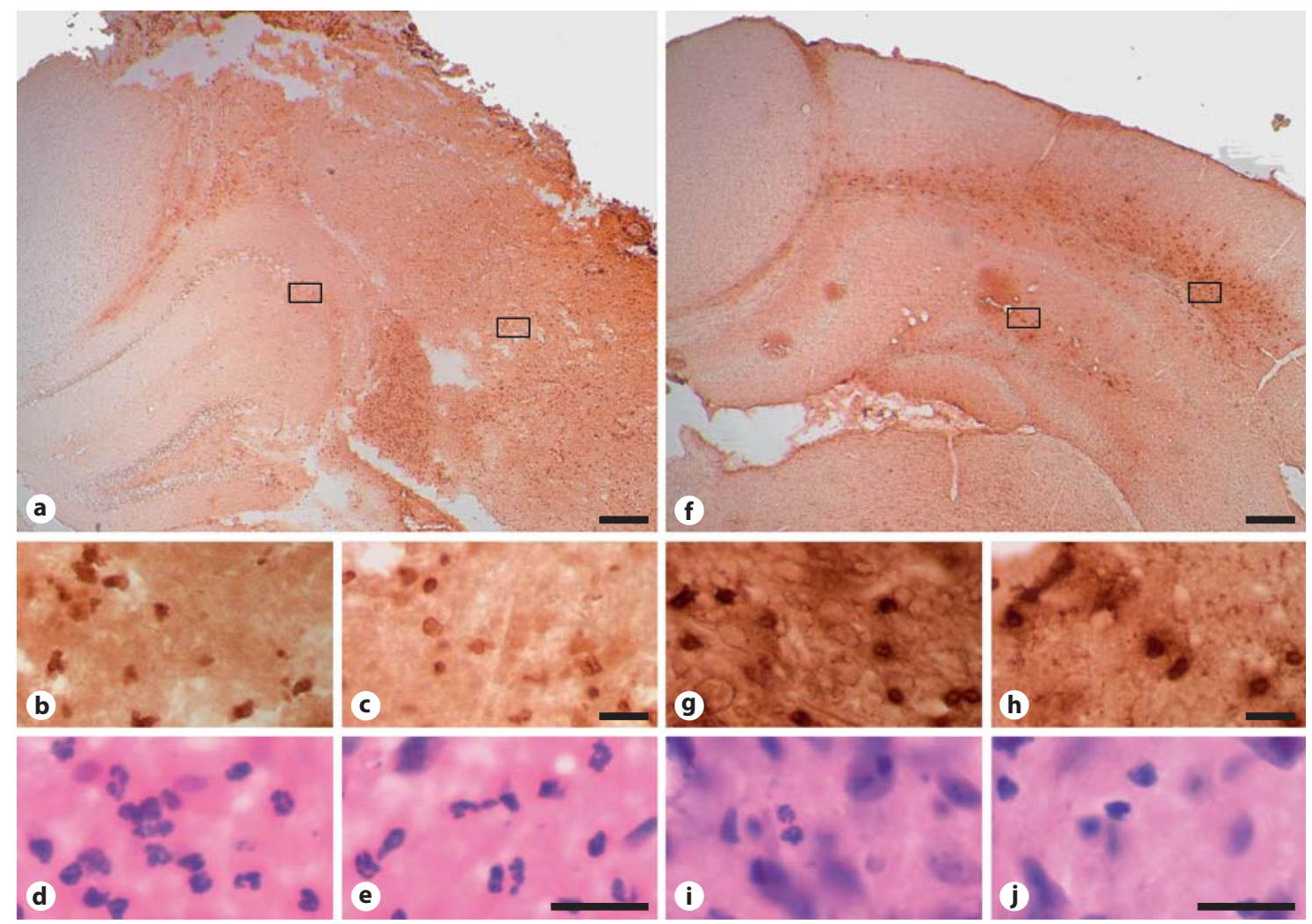

Fig. 3. GR-1+ neutrophils in the injured P21 brain. A granulocytic marker, GR-1, is localized in the P21 brain at 1 day (a-e) and 14 days $(\mathbf{f}-\mathbf{j})$ after injury in all animals. Note the prominence of these cells at both time points in the cortex and hippocampus of both groups. Boxes: location of enlarged areas of the cortex (b, g) and hippocampus $(\mathbf{c}, \mathbf{h})$. Adjacent sections stained with hematoxylin and eosin show cells with lobulated nuclei in the cortex (d, i) and hippocampus $(\mathbf{e}, \mathbf{j})$. Scale bars $=200 \mu \mathrm{m}(\mathbf{a}, \mathbf{f})$ and $20 \mu \mathrm{m}(\mathbf{c}, \mathbf{e}, \mathbf{h}, \mathbf{j})$.

CD45+, GR-1+, CD4+ and CD8+ cells were quantified within the injured cortex (fig. 5). To make the statistical assumptions of normality and equal variances, analyses were performed on square-root-transformed data. We first determined those time points in which the number of inflammatory cells in the ipsilateral cortex differed from that of the sham values, based upon one-way ANOVA and, where appropriate, Newman-Keuls multiple comparison post hoc tests. In the injured P21 brains, CD45+ and GR-1+ cell numbers were significantly higher within the first 2 weeks after injury $(\mathrm{p}<0.05)$, peaking at 1-3 days, and were not statistically distinguishable from the sham values by 35 days (fig. $5 \mathrm{a}, \mathrm{b}$ ). CD $4+$ cells peaked at 1 day after injury $(\mathrm{p}<0.001)$ and remained elevated over the first week $(\mathrm{p}<0.01)$ (fig. $5 \mathrm{c})$, whereas $\mathrm{CD} 8+$ cells were elevated early (at 3 days; $\mathrm{p}<0.001$ ) after injury and again during wound healing (at 14 days; $\mathrm{p}<$ 0.01 ) (fig. $5 \mathrm{~d}$ ). In the injured adult brains, the numbers of
CD45+ and GR-1+ cells in the cortex were elevated at 1 day (GR-1; p < 0.05) and 3 days after injury (CD45, p < 0.001; GR-1, $p<0.05$ ), but returned to the sham values thereafter (fig. $5 \mathrm{a}, \mathrm{b})$. CD4+ cells peaked 1 day after injury $(p<0.001)$ and remained elevated above control values for the first week $(\mathrm{p}<0.05)$ (fig. $5 \mathrm{c})$, whereas CD8+ cells were elevated at 3 and 14 days after injury $(\mathrm{p}<0.001$ and $\mathrm{p}<0.05$, respectively) (fig. $5 \mathrm{~d}$ ).

Next we examined the relationship between age at time of injury and time after injury. Based upon two-way ANOVA, the trajectories were not significantly different between the 2 age groups for CD $45+$ cells. Main effects analysis showed that, on average, the values of CD45 in the P21 group were significantly higher $(\mathrm{p}=0.001)$ than in the adult group. A similar trend was seen for GR-1 $(p=0.056)$. In contrast, no differences were noted for CD4 and CD8. These findings demonstrate age-related differences in infiltration of the panleukocyte marker 

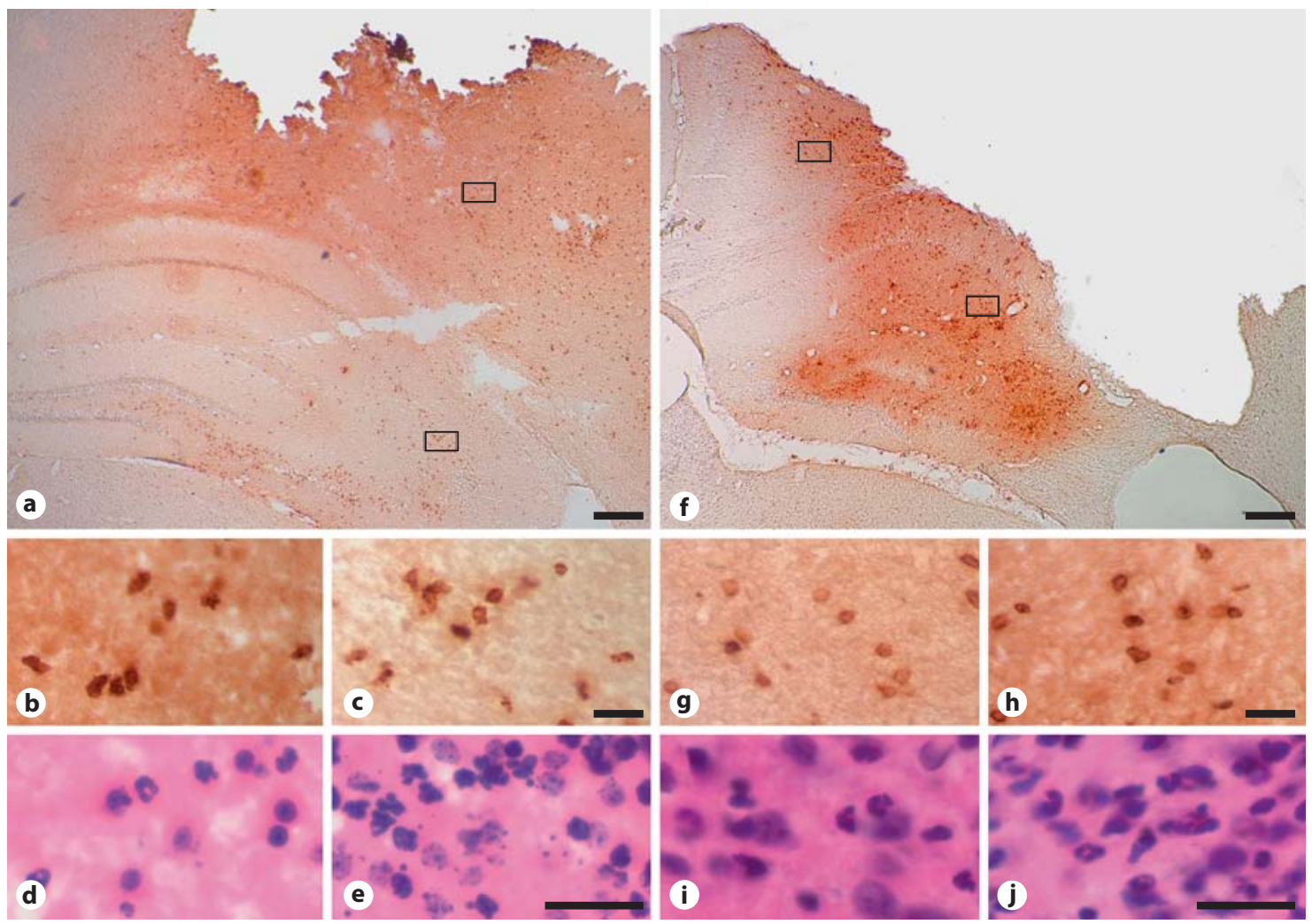

Fig. 4. GR-1+ neutrophils in the injured adult brain. GR-1+ cells are identified in the adult brain at 1 day (a-e), and in some animals, at 14 days $(\mathbf{f}-\mathbf{j})$ after injury in the cortex $(\mathbf{b}, \mathbf{g})$ and hippocampus $(\mathbf{c}, \mathbf{h})$. Boxes: location of enlarged areas of the cortex $(\mathbf{b}, \mathbf{g})$ and hippocampus $(\mathbf{c}, \mathbf{h})$. Based upon staining with hematoxylin and eosin, neutrophils are confirmed by their lobulated nuclei at both time points and in the cortex $(\mathbf{d}, \mathbf{i})$ and hippocampus $(\mathbf{e}, \mathbf{j})$. Scale bars $=200 \mu \mathrm{m}(\mathbf{a}, \mathbf{f})$ and $20 \mu \mathrm{m}(\mathbf{c}, \mathbf{e}, \mathbf{h}, \mathbf{j})$.

CD45 and suggest that these differences are primarily attributed to a subset of CD45+ leukocytes, namely GR-1+ granulocytes.

\section{Temporal Pattern of Irreversibly Damaged Cells in the}

Cortex Is Similar between Age Groups

The time course of irreversibly injured, TUNEL+ cells was evaluated in the cortex within each of the age groups at 1, 3 and 7 days after injury (fig. 5e). In both age groups, the number of TUNEL+ cells in the cortical mantle was significantly elevated at 1 day after injury (one-way ANOVA; $\mathrm{p}<0.01$ ), returning to baseline thereafter. Based upon two-way ANOVA, the trajectories of TUNEL+ cells were indistinguishable between the age groups. Main effects analysis revealed no differences. These data suggest that age is not a determinant of early patterns of cortical cell injury.

Age, Leukocyte Infiltration and Cortical Volume Loss after TBI
The Kinetics of Cortical Volume Loss Are Age Dependent

Cortical volume was analyzed at both 2 and 5 weeks after injury in each age group (fig. 6). Cortical volumes from sham-operated animals were similar at 2 and 5 weeks after surgery within each of the age groups.

The P21 brain-injured group showed a significant reduction in cortical volume to $79.69 \%$ of the sham-operated controls at 14 days after injury, and to $60.19 \%$ by 5 weeks. Importantly, there was a significant difference in cortical volumes between 2 and 5 weeks after injury (unpaired $t$ test; $p<0.05$ ). The adult brain-injured group also showed a reduction in cortical mantle volume to $50.68 \%$ of the sham-operated controls at 14 days after injury, and to $46.90 \%$ by 5 weeks. However, unlike in the injured P21 brains, there was no difference in cortical volumes between 2 and 5 weeks after injury (unpaired $t$ test; $p=$ $0.948)$.

Dev Neurosci 2010;32:454-465 

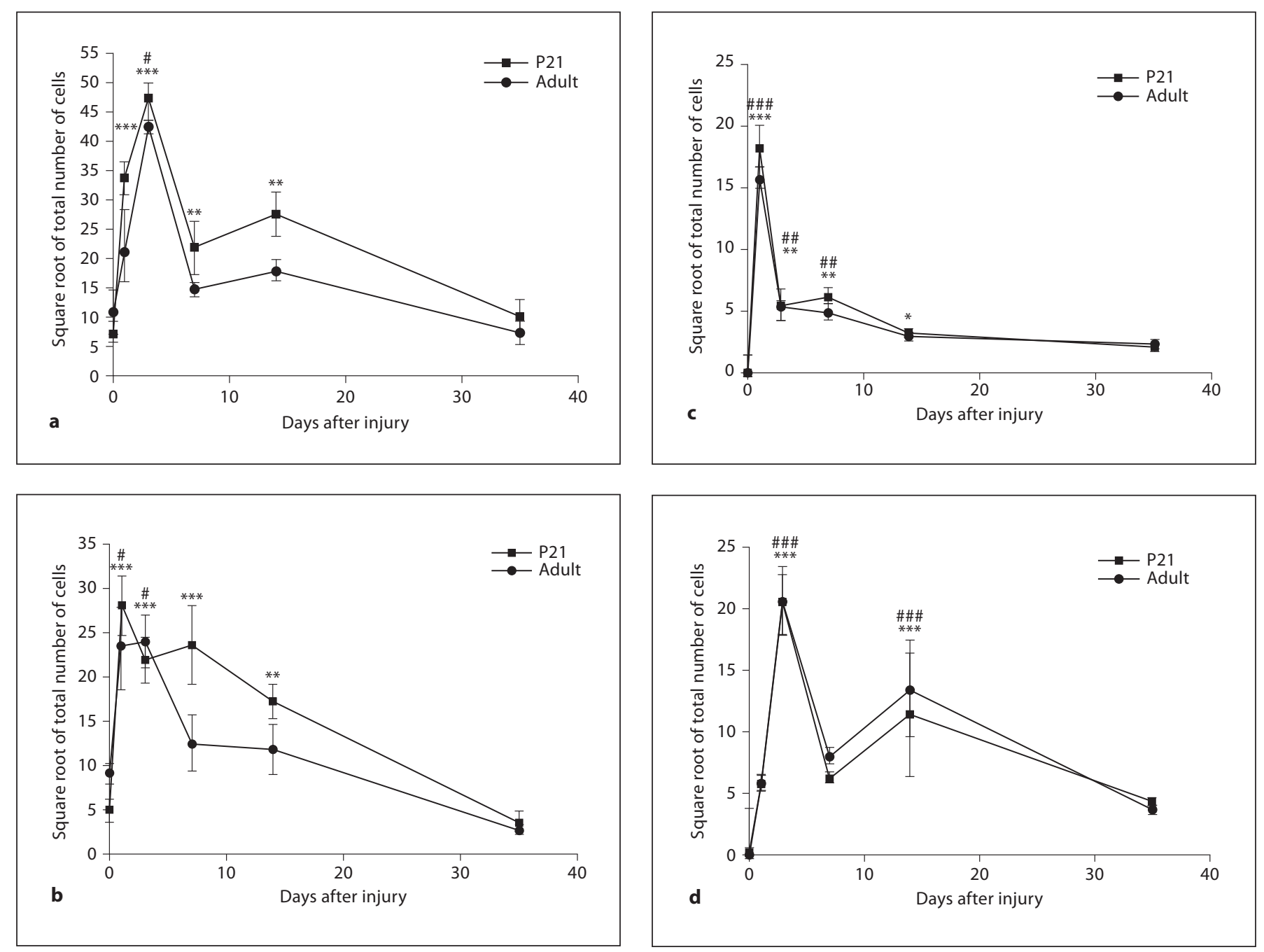

Fig. 5. Quantification of inflammatory cells in cortex after TBI. ${ }^{*} \mathrm{p}<0.05,{ }^{* *} \mathrm{p}<0.01,{ }^{* * *} \mathrm{p}<0.001$, one-way ANOVA followed by Newman-Keuls multiple comparison test (sham vs. P21); ${ }^{\#} \mathrm{p}<$ $0.05,{ }^{\# \#} \mathrm{p}<0.01,{ }^{\# \#} \mathrm{p}<0.001$, one-way ANOVA followed by Newman-Keuls multiple comparison test (sham vs. adult). a, b In the injured P21 brains, CD45 (a) and GR-1 (b) are significantly higher within the first 2 weeks after injury relative to shams. In the injured adult brains, CD45 is elevated at 3 days and GR-1 is elevated at 1 and 3 days after injury. c, d In the injured P21 brains, the number of CD4+ cells peaks at 1 day after injury and remains elevated at 14 days. In the injured adult brains, CD4 remains elevated over the first week. CD8 is elevated at 3 and 14 days after injury in both age groups. e TUNEL+ cells quantified within the cortex. In both age groups, the number of TUNEL+ cells in the cortical mantle is significantly elevated at 1 day after injury.

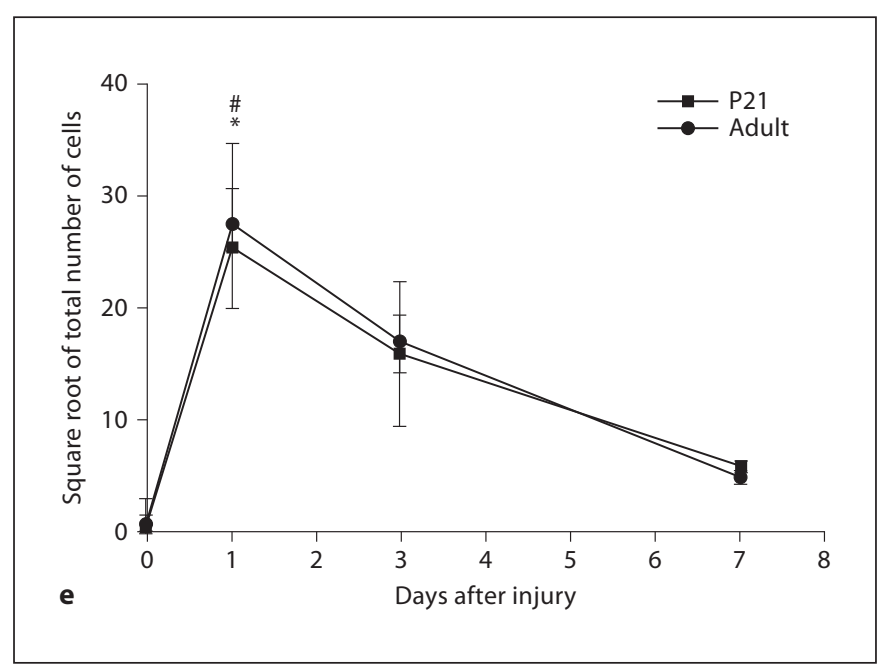




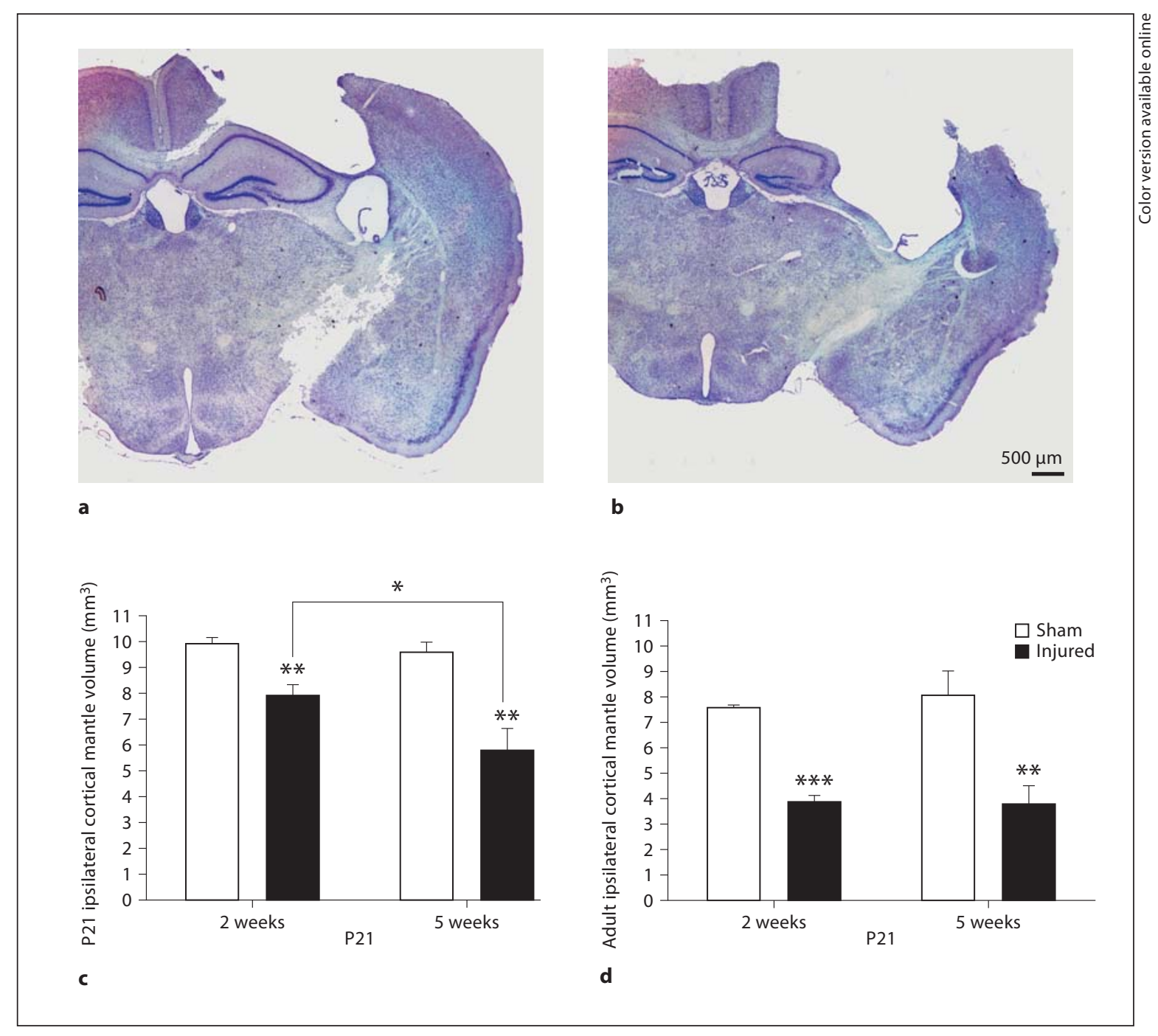

Fig. 6. Age-dependent reduction in cortical volume after TBI. a, b Typical appearance of a coronal section stained with cresyl violet in each age group at 5 weeks after injury. A cavitation is apparent in the ipsilateral cortex in both the P21 (a) and adult (b) brain. c, d The ipsilateral cortical mantle volume was determined in cresyl-violet-stained sections. Injury leads to a significant reduction in cortical mantle volume at 2 and 5 weeks after injury, as compared to sham-operated controls, in both the P21 (c) and adult groups (d). In the injured P21 group, there is a significant reduction in cortical mantle volume between 2 and 5 weeks. ${ }^{*} \mathrm{p}<0.05$, ${ }^{* *} \mathrm{p}<0.01,{ }^{* * *} \mathrm{p}<0.001$, unpaired $\mathrm{t}$ test.
We next compared the injured ipsilateral cortical volumes, as ratios to their respective shams, between the $\mathrm{P} 21$ and adult groups for each time point. At 2 weeks after injury, the cortical volume of the adult group was significantly reduced relative to the $\mathrm{P} 21$ group (unpaired t test; $\mathrm{p}<0.01$ ). However, by 5 weeks after injury, there was no difference between the 2 groups (unpaired t test; $\mathrm{p}=$ 0.316). Together, these findings suggest age-related, temporal differences in long-term loss of cortical volume. Whereas the injured adult brain undergoes a more accel- erated loss within the first 2 weeks after injury, the developing brain is characterized by a more gradual long-term reduction in cortical volume.

\section{Discussion}

Here we hypothesized age-dependent differences in the inflammatory response and expansion of the cortical lesion after TBI. To test this hypothesis, we profiled leu- 
kocyte infiltration and changes in ipsilateral cortical volume in the developing and adult brain after TBI. Several interesting findings have emerged from this effort. The assessment of early cerebral blood flow and cortical cell injury over the first week after injury revealed no differences between the age groups. However, the magnitude and temporal patterns of leukocyte infiltration and loss of cortical volume were age dependent. The injured P21 brain showed greater numbers of CD45+ and GR-1+ leukocytes in the cortex over the course of several weeks. Whereas both the P21 and adult brains showed marked reductions in cortical volume between 2 and 5 weeks after injury, the kinetics of this reduction were different. There was a greater loss of cortical volume by 2 weeks after injury in the adult brain with no further reductions thereafter. In contrast, the P21 brain showed a more modest early reduction in cortical volume that reached values similar to the adult brain by 5 weeks after injury. Together, these findings highlight unique differences in how the developing and adult brains respond to TBI. Both leukocyte infiltration and loss of cortical volume, most likely related to the expansion of the cortical lesion, occur over a longer time period than in the injured adult brain. As TBI to the developing brain results in cognitive deficits that emerge during wound healing [Pullela et al., 2006], further studies will be needed to address the mechanisms underlying these age-related differences and the extent to which they contribute to cognitive decline during brain maturation.

\section{Relative Blood Flow after TBI}

In this study, we profiled the time course of blood flow after injury to the developing and adult brains. Cerebral blood flow was depressed in both groups immediately after injury. Surprisingly, we also found that blood flow dramatically increased in both groups by at least 7 days after injury and remained elevated up to 28 days thereafter. While factors contributing to the increase in flow remain unclear, nitric oxide (NO) may direct this hyperemia.

NO is a cell-membrane-permeant, free radical gas with diverse functions including the maintenance of basal vasomotor tone (see reviews in Cherian et al. [2004], and Moncada and Higgs [2006]) that is synthesized from L-arginine by nitric oxide synthase (NOS), which has three isoforms: neuronal (nNOS), endothelial (eNOS) and inducible (iNOS). Several lines of evidence support the hypothesis that iNOS may be a key mediator of hyperemia. iNOS is induced in both the injured developing [Clark et al., 1996] and adult brain [Wada et al., 1998] in vascular smooth muscle and infiltrating neutrophils [Clark et al., 1996] as well as in macrophages and astrocytes over the course of the first week after injury [Wada et al., 1998], and parallels the timing of hyperemia seen in the current study. Finally, posttraumatic hyperemia seen in the adult wild type is attenuated in iNOS knockout mice, a finding that further reinforces the role of NO in the modulation of cerebral blood flow [Foley et al., 2008].

\section{TUNEL and the Injured Brain}

In both the P21 and adult-aged groups, TUNEL staining was maximal at 1 day and declined thereafter to control values within the first week after injury. Interestingly, this pattern of cell damage corresponds to the elevated numbers of GR-1+ neutrophils within the injured cortical mantle in both age groups. Whether infiltrated neutrophils contribute to this cell injury is not clear. Indirect evidence would support their participation in pathogenesis, particularly as it relates to barrier function and edema formation. For example, in the injured adult brain, the recruitment of leukocytes [Schoettle et al., 1990; Biagas et al., 1992; Soares et al., 1995; Hartl et al., 1997] coincides with a disruption of the blood-brain barrier [Soares et al., 1995]. Strategies to increase circulating neutrophils result in increased permeability of the bloodbrain barrier after TBI [Whalen et al., 2000]. There have been only few studies that have addressed the role of neutrophils in the developing brain after injury. In a model of neonatal hypoxia-ischemia, there was reduced brain swelling in animals that have been made neutropenic by antineutrophil serum, or that have been treated with allopurinol [Hudome et al., 1997].

\section{Leukocyte Infiltration after TBI}

This study shows that infiltration of leukocytes, as measured by an antibody to the panleukocytic marker $\mathrm{CD} 45$, is increased following injury, and that there is greater leukocyte infiltration in the developing as compared to the adult brain. Similar kinetics of infiltration are seen with an independent assessment of GR-1+ leukocytes, a subset of the CD45 population that are neutrophils based upon their classic lobulated nuclei. This agedependent difference is not a generic response to all leukocytes, as the kinetics of CD4+ and CD8+ T cells were similar between age groups.

Our findings of age-dependent differences are consistent with others; adult mice are more resistant to inflammation, as evidenced by less leukocyte recruitment in response to cytokines, than 1- to 3-week-old mice [Lawson 
and Perry, 1995; Anthony et al., 1997]. This has led to the hypothesis that there is a 'window of susceptibility' where the developing brain is less refractory to inflammatory stimuli than the adult brain [Lawson and Perry, 1995; Anthony et al., 1997; Campbell et al., 2002]. The biologic basis for this age-related 'window of susceptibility' is not clear. Early studies addressed age-related differences in the context of chemokine responses. Macrophage inflammatory protein-2, a CXC chemokine that induces neutrophil chemotaxis, is more rapidly induced in the young brain than the adult brain after intraparenchymal administration of cytokines [Anthony et al., 1998]. Neutrophil infiltration, which parallels this induction, is likewise greater in the young brain. Together, these findings suggest that marked neutrophil infiltration, seen in the young brain, may reflect increased susceptibility to the neutrophil chemoattractive effects of CXC chemokines [Anthony et al., 1998].

It is of interest that while CD45+/GR-1+ cells peak at $1-3$ days after injury to the developing brain, they remain elevated above controls for the next several weeks. To our knowledge there are no studies that have addressed trafficking of neutrophils beyond the acute period after TBI. However, in a murine experimental model of spinal cord injury, neutrophils are present beginning at $6 \mathrm{~h}$ and extending up to 6 weeks later [Kigerl et al., 2006]. Such findings suggest that there is a prolonged period of neutrophil infiltration and/or that the mechanisms modulating neutrophil apoptosis are altered. While cytokines, including those that function as neutrophil chemoattractants (CXCL1, CXCl2, CXC8) [Semple et al., 2010b] may contribute to long-term neutrophil transmigration into the injured brain, it is also possible that an imbalance between pro- and antiapoptotic mechanisms could translate into increased survival of neutrophils [Kigerl et al., 2006].

\section{Age-Dependent Loss of Cortical Volume}

We find that the kinetics of loss of cortical mantle volume after TBI are age dependent. Whereas a marked reduction in ipsilateral cortical mantle volume is seen in the adult brain by 2 weeks after injury, there is a relatively more gradual loss of cortical volume in the injured P21 brain, reaching values similar to that seen in the injured adult brain by 5 weeks after injury.

Loss of cortical volume most likely reflects the expansion of the cortical lesion. An enlarging cortical lesion has been reported in the adult rodent within the first several weeks after injury [Yoneyama-Sarnecky et al., 2010], with continued expansion up to 1 year after injury [Smith et al., 1997]. In the developing brain, there is likewise a significant expansion of the cortical lesion occurring between 2 weeks and 3 months after injury [Pullela et al., 2006]. Of interest, this expanding lesion in the P21 brain corresponds to emerging cognitive deficits [Pullela et al., 2006].

Antioxidant status and a proinflammatory state are candidate determinants of the expanding cortical lesion and the resultant loss of cortical volume. In the injured adult brain, the expansion of the cortical lesion is accelerated within the first several weeks after TBI to adult mice deficient in heme oxygenase 2, an enzyme that plays a key role in the metabolism of the prooxidant heme [Yoneyama-Sarnecky et al., 2010]. Such a finding suggests that this enzyme, ubiquitously expressed throughout the CNS [Maines et al., 1996; Ewing and Maines, 1997], provides an endogenous line of defense against heme-directed pathogenesis.

Glutathione peroxidase (GPx), an antioxidant that metabolizes hydrogen peroxide to water and oxygen, has been shown to be neuroprotective to the developing brain. Immature neurons, cultured from GPx-overexpressing transgenic ( $\mathrm{Tg}$ ) mice, show less injury when exposed to hydrogen peroxide. Moreover, susceptibility to neonatal hypoxic-ischemic injury is reduced in the GPxoverexpressing Tg animal. Such findings have prompted further study of GPx in the setting of TBI at P21. While cortical volumes, measured when these animals reached adulthood, were reduced in both the $\mathrm{Tg}$ and wild-type groups, there was no effect of genotype [Tsuru-Aoyagi et al., 2009]. Such findings suggest that other factors insensitive to GPx activity, such as ongoing secondary pathogenesis and/or aberrant developmental processes, may contribute to cortical volume loss.

A key question remains with regard to the age-dependent factors that govern the loss of cortical volume and expansion of the cortical lesion. Some insight may be gained from studies of the CC ligand-2 (CCL2) knockout animal [Semple et al., 2010a]. CCL2 is a chemokine involved in macrophage recruitment. Brain-injured mice, deficient in CCL2, show a reduction in lesion volume and a reduced spread of F4/80 macrophages in the damaged cortex. However, these differences are not detected within the first week after injury, but rather become evident between 2 and 4 weeks after injury. It is hypothesized that this response may reflect the more delayed recruitment of hematogenous macrophages to the injured brain [Semple et al., 2010a]. With this hypothesis in mind, it is conceivable that age-related differences in lesion expansion, seen in the current study, may be governed by the magni- 
tude of infiltration of hematogenous macrophages, with initially fewer of these macrophages recruited to the injured developing brain relative to the adult brain.

\section{Conclusion}

In conclusion, we demonstrate age-dependent differences in the kinetics of infiltration of a subset of leukocytes and loss of cortical mantle volume following TBI. Understanding the factors that contribute to these differ- ences will be a critical first step in explaining the progression of pathogenesis following childhood injury, and eventually in developing age-appropriate therapeutics.

\section{Acknowledgments}

The authors thank Seong E. Koh (MD) and Hovhannes Manvelyan (MD) for their contributions to this project. The study was supported by NIH/NINDS grants No. R01NS050159 and R21NS065937.

\section{References}

Anthony DC, Bolton SJ, Fearn S, Perry VH (1997): Age-related effects of interleukin-1 beta on polymorphonuclear neutrophil-dependent increases in blood-brain barrier permeability in rats. Brain 120(pt 3):435444.

Anthony DC, Dempster R, Fearn S, Clements J, Wells G, Perry VH, Walker K (1998): CXC chemokines generate age-related increases in neutrophil-mediated brain inflammation and blood-brain barrier breakdown. Curr Biol 8:923-926.

Biagas KV, Uhl MW, Schiding JK, Nemoto EM, Kochanek PM (1992): Assessment of posttraumatic polymorphonuclear leukocyte accumulation in rat brain using tissue myeloperoxidase assay and vinblastine treatment. J Neurotrauma 9:363-371.

Campbell SJ, Wilcockson DC, Butchart AG, Perry VH, Anthony DC (2002): Altered chemokine expression in the spinal cord and brain contributes to differential interleukin- $1 \beta$ induced neutrophil recruitment. J Neurochem 83:432-441.

Cherian L, Hlatky R, Robertson CS (2004): Nitric oxide in traumatic brain injury. Brain Pathol 14:195-201.

- Clark RS, Kochanek PM, Schwarz MA, Schiding JK, Turner DS, Chen M, Carlos TM, Watkins SC (1996): Inducible nitric oxide synthase expression in cerebrovascular smooth muscle and neutrophils after traumatic brain injury in immature rats. Pediatr Res 39:784790.

Ditelberg JS, Sheldon RA, Epstein CJ, Ferriero DM (1996): Brain injury after perinatal hypoxia-ischemia is exacerbated in copper/zinc superoxide dismutase transgenic mice. Pediatr Res 39:204-208.

-Dixon CE, Clifton GL, Lighthall JW, Yaghmai AA, Hayes RL (1991): A controlled cortical impact model of traumatic brain injury in the rat. J Neurosci Methods 39:253-262.
Durkin MS, Olsen S, Barlow B, Virella A, Connolly ES Jr (1998): The epidemiology of urban pediatric neurological trauma: evaluation of, and implications for, injury prevention programs. Neurosurgery 42:300-310.

Ewing JF, Maines MD (1997): Histochemical localization of heme oxygenase- 2 protein and mRNA expression in rat brain. Brain Res Brain Res Protoc 1:165-174.

Fan P, Yamauchi T, Noble LJ, Ferriero DM (2003): Age-dependent differences in glutathione peroxidase activity after traumatic brain injury. J Neurotrauma 20:437-445.

-Ferriero DM (2001): Oxidant mechanisms in neonatal hypoxia-ischemia. Dev Neurosci 23:198-202.

Fife D, Jagger J (1984): The contribution of brain injury to the overall injury severity of braininjured patients. J Neurosurg 60:697-699.

Foley LM, Hitchens TK, Melick JA, Bayir H, Ho C, Kochanek PM (2008): Effect of inducible nitric oxide synthase on cerebral blood flow after experimental traumatic brain injury in mice. J Neurotrauma 25:299-310.

Frankowski RF (1986): Descriptive epidemiologic studies of head injury in the United States: 1974-1984. Adv Psychosom Med 16:153172.

Gundersen HJ, Jensen EB (1987): The efficiency of systematic sampling in stereology and its prediction. J Microsc 147(pt 3):229-263.

-Hartl R, Medary MB, Ruge M, Arfors KE, Ghajar J (1997): Early white blood cell dynamics after traumatic brain injury: effects on the cerebral microcirculation. J Cereb Blood Flow Metab 17:1210-1220.

Hudome S, Palmer C, Roberts RL, Mauger D, Housman C, Towfighi J (1997): The role of neutrophils in the production of hypoxicischemic brain injury in the neonatal rat. Pediatr Res 41:607-616.

Igarashi T, Huang TT, Noble LJ (2001): Regional vulnerability after traumatic brain injury: gender differences in mice that overexpress human copper, zinc superoxide dismutase. Exp Neurol 172:332-341.
Kaczorowski SL, Schiding JK, Toth CA, Kochanek PM (1995): Effect of soluble complement receptor-1 on neutrophil accumulation after traumatic brain injury in rats. J Cereb Blood Flow Metab 15:860-864.

Kawabata K, Hagio T, Matsuoka S (2002): The role of neutrophil elastase in acute lung injury. Eur J Pharmacol 451:1-10.

Kigerl KA, McGaughy VM, Popovich PG (2006): Comparative analysis of lesion development and intraspinal inflammation in four strains of mice following spinal contusion injury. J Comp Neurol 494:578-594.

Kraus JF, Rock A, Hemyari P (1990): Brain injuries among infants, children, adolescents, and young adults. Am J Dis Child 144:684691.

Lawson LJ, Perry VH (1995): The unique characteristics of inflammatory responses in mouse brain are acquired during postnatal development. Eur J Neurosci 7:1584-1595.

Lee W, Downey G (2001): Leukocyte elastase: physiological functions and role in acute lung injury. Am J Respir Crit Care Med 164: 896-904.

Levin HS, Eisenberg HM, Wigg NR, Kobayashi K (1982): Memory and intellectual ability after head injury in children and adolescents. Neurosurgery 11:668-673.

-Luerssen TG, Klauber MR, Marshall LF (1988): Outcome from head injury related to patient's age: a longitudinal prospective study of adult and pediatric head injury. J Neurosurg 68:409-416.

Maines MD, Eke BC, Zhao X (1996): Corticosterone promotes increased heme oxygenase- 2 protein and transcript expression in the newborn rat brain. Brain Res 722:83-94.

Mischel RE, Kim YS, Sheldon RA, Ferriero DM (1997): Hydrogen peroxide is selectively toxic to immature murine neurons in vitro. Neurosci Lett 231:17-20.

Moncada S, Higgs EA (2006): The discovery of nitric oxide and its role in vascular biology. Br J Pharmacol 147(suppl 1):S193-S201. 
Owen C, Campbell E (1999): The cell biology of leukocyte-mediated proteolysis. J Leukoc Biol 65:137-150.

Paxinos G, Watson C (1986): The Rat Brain in Stereotaxic Coordinates. San Diego, Academic Press.

- Philip S, Udomphorn Y, Kirkham FJ, Vavilala MS (2009): Cerebrovascular pathophysiology in pediatric traumatic brain injury. J Trauma 67:S128-S134.

- Pullela R, Raber J, Pfankuch T, Ferriero DM, Claus CP, Koh SE, Yamauchi T, Rola R, Fike JR, Noble-Haeusslein LJ (2006): Traumatic injury to the immature brain results in progressive neuronal loss, hyperactivity and delayed cognitive impairments. Dev Neurosci 28:396-409.

Reed MG, Howard CV (1998): Surface-weighted star volume: concept and estimation. J Microsc 190:350-356.

- Schoettle RJ, Kochanek PM, Magargee MJ, Uhl MW, Nemoto EM (1990): Early polymorphonuclear leukocyte accumulation correlates with the development of posttraumatic cerebral edema in rats. J Neurotrauma 7:207-217.
Semple BD, Bye N, Rancan M, Ziebell JM, Morganti-Kossmann MC (2010a): Role of CCL2 (MCP-1) in traumatic brain injury (TBI): evidence from severe TBI patients and CCL2-/- mice. J Cereb Blood Flow Metab 30: 769-782.

-Semple BD, Kossmann T, Morganti-Kossmann MC (2010b): Role of chemokines in CNS health and pathology: a focus on the CCL2/ CCR2 and CXCL8/CXCR2 networks. J Cereb Blood Flow Metab 30:459-473.

Smith DH, Chen XH, Pierce JE, Wolf JA, Trojanowski JQ, Graham DI, McIntosh TK (1997): Progressive atrophy and neuron death for one year following brain trauma in the rat. J Neurotrauma 14:715-727.

-Soares HD, Hicks RR, Smith D, McIntosh TK (1995): Inflammatory leukocytic recruitment and diffuse neuronal degeneration are separate pathological processes resulting from traumatic brain injury. J Neurosci 15 8223-8233.

Tong W, Igarashi T, Ferriero DM, Noble LJ (2002): Traumatic brain injury in the immature mouse brain: characterization of regional vulnerability. Exp Neurol 176:105116.
Tsuru-Aoyagi K, Potts MB, Trivedi A, Pfankuch T, Raber J, Wendland M, Claus CP, Koh SE, Ferriero D, Noble-Haeusslein LJ (2009): Glutathione peroxidase activity modulates recovery in the injured immature brain. Ann Neurol 65:540-549.

Wada K, Chatzipanteli K, Kraydieh S, Busto R, Dietrich WD (1998): Inducible nitric oxide synthase expression after traumatic brain injury and neuroprotection with aminoguanidine treatment in rats. Neurosurgery 43: 1427-1436.

Whalen MJ, Carlos TM, Wisniewski SR, Clark RS, Mellick JA, Marion DW, Kochanek PM (2000): Effect of neutropenia and granulocyte colony stimulating factor-induced neutrophilia on blood-brain barrier permeability and brain edema after traumatic brain injury in rats. Crit Care Med 28:3710-3717.

Yoneyama-Sarnecky T, Olivas A, Azari S, Ferriero D, Manvelyan $\mathrm{H}$, Noble-Haeusslein L (2010): Heme oxygenase-2 modulates early pathogenesis after traumatic injury to the immature brain. Dev Neurosci 32:81-90.

\section{Erratum}

Please note that the authorship of the article 'Age Is a Determinant of Leukocyte Infiltration and Loss of Cortical Volume after Traumatic Brain Injury', Dev Neurosci 2010;32:454-465, DOI:10.1159/000316805, published online: September 15, 2010, should read as follows:

\section{Catherine P. Claus $^{\mathrm{a}}$ Kyoko Tsuru-Aoyagi ${ }^{\mathrm{a}}$ Hita Adwanikar ${ }^{\mathrm{a}}$ Breset Walker $^{b}$ Hovhannes Manvelyan $^{d}$ William Whetstone $^{b}$ Linda J. Noble-Haeusslein ${ }^{a, c}$}

Departments of a Neurosurgery, ${ }^{b}$ Emergency Medicine, ${ }^{c}$ Physical Therapy and Rehabilitation Science, University of California, San Francisco, Calif., USA; ${ }^{\mathrm{d}}$ Department of Neurology, Yerevan State Medical University, Yerevan, Armenia

Accordingly, the Acknowledgments should read:

The authors thank Seong E. Koh, MD, for his contributions to this project. The study was supported by NIH/NINDS grants No. R01NS050159 and R21NS065937. 